\title{
On Lebesgue measure and Hausdorff dimension of Julia sets of real periodic points of renormalization
}

by

\author{
Artem DUDKO
}

\section{Presented by Feliks PRZYTYCKI}

Summary. We give a new sufficient condition for the Julia set of a real analytic function which is a periodic point of renormalization to have Hausdorff dimension less than 2. This condition can be verified numerically. We present results of computer experiments suggesting that this condition is satisfied for real periodic points of renormalization with low periods. Our results support the conjecture that all real Feigenbaum maps have Julia sets of Hausdorff dimension less than 2.

Informally speaking, the Julia set of a rational map on a complex plane is the set of points near which the dynamics induced by this map is chaotic, i.e. has unpredictable long-time behavior. A natural question is how large this set can be. The size of a subset of a complex plane can be measured using Lebesgue area or Hausdorff dimension. For a long time it was conjectured that for any rational map the Julia set either coincides with the whole plane or has zero area. Recently, Buff and Cheritat [4 found first examples of quadratic maps having Julia sets of positive measure. Later, Avila and Lyubich [2] constructed such examples in the class of maps whose Julia sets have stronger self-similar properties, called Feigenbaum maps. However, the constructions of those examples are quite involved and the search for simpler examples continues. For instance, an open question is whether there exist quadratic Feigenbaum maps with real coefficients having Julia sets of positive area, or at least Hausdorff dimension 2. Jointly with Sutherland, the

2020 Mathematics Subject Classification: Primary 37F25; Secondary 37F35.

Key words and phrases: renormalization, Feigenbaum maps, Julia set, Hausdorff dimension.

Received 6 April 2021.

Published online 20 April 2021. 
author of the present paper showed in [8] that the Julia set of the original quadratic Feigenbaum map (corresponding to the period-doubling renormalization) has Hausdorff dimension less than 2, and thus has zero area.

Here we generalize the approach of $[8]$ to an arbitrary real periodic point of renormalization. Given such a map $f$, the main result of this paper (Theorem 4 provides a sufficient condition for the Julia set of $f$ to be of Hausdorff dimension less than 2 . This condition can be checked effectively using a computer. Approximate estimates suggest that it is satisfied by periodic points of renormalization with low periods. These estimates support the following:

ConjeCture 1. Every real Feigenbaum quadratic map has Julia set of Hausdorff dimension less than 2 (and thus of zero Lebesgue area).

The paper is organized as follows. In Section 1 we recall first the notion of renormalization. In Subsection 1.1 we briefly explain the Avila-Lyubich approach to study measure-theoretic properties of Feigenbaum Julia sets. In Subsection 1.2 we recall some basic properties of real periodic points of renormalization and present the main result of the present paper. In Section 2 we discuss structural properties of real periodic points of renormalization. In particular, we introduce a certain tiling structure of the plane and study its properties. In Section 3 we prove the main result. First, in Subsection 3.1 we introduce copies of tiles under certain iterates of the Feigenbaum map. Then in Subsection 3.2 we study the distortion of these iterates on the corresponding copies. Finally, in Subsection 3.3 we prove analogues of Avila-Lyubich recursive inequalities and complete the proof of the main result. In Section 4 we present numerical results and discuss briefly some of the algorithms used to obtain these results.

1. Preliminaries. Let us briefly recall some notions from complex dynamics which we use. For details we refer the reader to [7], [17], or [20]. By definition, a quadratic-like map is a ramified covering $f: U \rightarrow V$ of degree 2, where $U \Subset V$ are topological disks in $\mathbb{C}$. Given a quadratic-like map $f$, its filled Julia set $K_{f}$ and Julia set $J_{f}$ are defined as follows:

$$
K_{f}=\left\{z \in U: f^{n}(z) \in U \text { for all } n \in \mathbb{N}\right\}, \quad J_{f}=\partial K_{f} .
$$

Let $f: U \rightarrow V$ be a quadratic-like map. Then $f$ is called renormalizable of period $n$ with $n>1$ if there exists $U^{\prime} \subset U$ for which $f^{n}: U^{\prime} \rightarrow V^{\prime}=f^{n}\left(U^{\prime}\right)$ is a quadratic-like map with connected Julia set $J^{\prime}$. The map $\left.f^{n}\right|_{U^{\prime}}$ is called a pre-renormalization of $f$; the map $\mathcal{R} f:=\left.\Lambda \circ f^{n}\right|_{U^{\prime}} \circ \Lambda^{-1}$, where $\Lambda$ is an appropriate rescaling of $U^{\prime}$, is called the renormalization of $f$.

An infinitely renormalizable quadratic-like map $f$ is called a Feigenbaum map if it has bounded combinatorics (i.e. there is a uniform bound on the periods $n$ of renormalization) and a priori bounds (the moduli of $V \backslash U$ 
are uniformly bounded). The first and one of the most studied Feigenbaum maps is the Feigenbaum polynomial $f_{\text {Feig }}(z)=z^{2}+c_{\text {Feig }}$, where $c_{\text {Feig }} \approx-1.4011551890$ is the limit of the sequence of real period doubling parameters. The discovery of $f_{\text {Feig }}$ and related Universality Phenomenon by Coullet, Tresser and Feigenbaum [6, 13, 14] led to the development of renormalization theory in dynamics.

The results of Sullivan imply that the sequence of successive perioddoubling renormalizations $\mathcal{R}^{n}\left(f_{\text {Feig }}\right)$ converge to a renormalization fixed point $F_{*}$ (see e.g. [19]). In a series of works by Lanford [16], Epstein [11] and [12, Sullivan [21, McMullen [20], and others it was shown that the renormalization operator $\mathcal{R}$ is hyperbolic near $F_{*}$. In fact, [20] and [21] generalized some of the properties of $F_{*}$ to other infinitely renormalizable maps. Lyubich [18] obtained a proof of hyperbolicity of $\mathcal{R}$ near infinitely renormalizable maps with bounded combinatorics (which includes periodic points of renormalization). We refer the reader to [18, §1.5], for a more detailed overview of the topic.

In [8] we showed with Sutherland that the Julia set of $F_{*}$ has Hausdorff dimension less than 2. The present paper concerns the measure and Hausdorff dimension of the Julia sets of the real periodic points of renormalization, i.e. Feigenbaum maps $f$ such that $f(z)=\sum_{k \geq 0} a_{k} z^{k}$ near zero with $a_{k} \in \mathbb{R}$ and $\mathcal{R}^{n} f=f$ for some $n$.

1.1. The Avila-Lyubich trichotomy. Consider a Feigenbaum map $f$. Let $f_{n}$ denote the $n$th pre-renormalization of $f$, let $J_{n}$ be its Julia set, and let $\mathcal{O}$ be the forward orbit of the critical orbit. For two sequences $\left\{a_{n}\right\},\left\{b_{n}\right\}$ of positive reals we will write $a_{n} \asymp b_{n}$ if there exists $C>0$ such that $C^{-1} b_{n}<a_{n}<C b_{n}$ for all $n$. To study the measure and Hausdorff dimension of the Julia sets of Feigenbaum maps Avila and Lyubich introduced so called nice domains (see [1] and [2]). These are domains $U^{n} \subset V^{n}$ satisfying a series of conditions:

- $f_{n}\left(U^{n}\right)=V^{n}$

- $U^{n} \supset J_{n} \cap \mathcal{O}(f)$;

- $V^{n+1} \subset U^{n}$;

- $f^{k}\left(\partial V^{n}\right) \cap V^{n}=\emptyset$ for all $n, k$;

- $A^{n}=V^{n} \backslash U^{n}$ is "far" from $\mathcal{O}(f)$;

- $\operatorname{area}\left(A^{n}\right) \asymp \operatorname{area}\left(U^{n}\right) \asymp \operatorname{diam}\left(U^{n}\right)^{2} \asymp \operatorname{diam}\left(V^{n}\right)^{2}$.

Avila and Lyubich showed that such domains exist for every Feigenbaum map. Notice that the construction of $U^{n}$ and $V^{n}$ involves cutting neighborhoods of the critical point by equipotentials and external rays of prerenormalizations $f_{n}$ of $f$ and taking preimages under long iterates of $f_{n}$, and it is computationally complex. 
For $n \in \mathbb{N}$ denote by $X_{n}$ the set of points in $U^{0}$ that land in $V^{n}$ under some iterate of $f$, and by $Y_{n}$ the set of points in $A^{n}$ that never return to $V^{n}$ under iterates of $f$ :

$$
\begin{aligned}
X_{n} & =\left\{z \in U^{0}: f^{k}(z) \in V^{n} \text { for some } k \in \mathbb{Z}_{+}\right\}, \\
Y_{n} & =\left\{z \in A^{n}: f^{k}(z) \notin V^{n} \text { for all } k \in \mathbb{N}\right\} .
\end{aligned}
$$

Introduce the quantities

$$
\eta_{n}=\frac{\operatorname{area}\left(X_{n}\right)}{\operatorname{area}\left(U^{0}\right)}, \quad \xi_{n}=\frac{\operatorname{area}\left(Y_{n}\right)}{\operatorname{area}\left(A^{n}\right)} .
$$

An important tool in the Avila-Lyubich approach to study the measure and Hausdorff dimension of Feigenbaum Julia sets is the following (see [1]):

TheOREM 2 (Avila-Lyubich). Let $f$ be a periodic point of renormalization, i.e. there is a $p$ such that $\mathcal{R}^{p} f=f$. Then exactly one of the following is true:

LEAN CASE: $\eta_{n}$ converges to 0 exponentially fast, $\inf \xi_{n}>0$, and $\operatorname{dim}_{\mathrm{H}}\left(J_{f}\right)$ $<2$;

BALANCED CASE: $\eta_{n} \asymp \xi_{n} \asymp 1 / n$ and $\operatorname{dim}_{H}\left(J_{f}\right)=2$ with area $\left(J_{f}\right)=0$; BLACK HOLE CASE: inf $\eta_{n}>0, \xi_{n}$ converges to 0 exponentially fast, and $\operatorname{area}\left(J_{f}\right)>0$.

Specific bounds determining the behavior of $\eta_{n}$ and $\xi_{n}$ depend on the geometry of $A^{n}$ and $\mathcal{O}(f)$.

The proof of Theorem 2 relies on recursive estimates involving $\eta_{n}, \xi_{n}$, and the Poincaré series for $f_{n}$. In the Lean case (relevant for this paper), Avila and Lyubich showed existence of a constant $C>0$ which only depends on geometric bounds for $U^{n} \subset V^{n}$ and $\mathcal{O}(f)$, such that if there exists $m$ with $\eta_{m}<\xi_{m} / C$, then $\eta_{n} \rightarrow 0$ exponentially fast. However, their proof is non-constructive and does not provide any way of estimating $C$ numerically. In this paper, we develop a recursive scheme for real periodic points of renormalization similar to Avila-Lyubich's but such that all objects can be effectively estimated using a computer.

1.2. Real periodic points of renormalization and main results. Fix a real periodic point $F$ of the quadratic-like renormalization: $\mathcal{R}^{s}(F)=F$. In this paper we do not require the period of renormalization to be minimal possible. This allows us to view $F$ as a fixed point of a renormalization with a longer period. We normalize $F$ so that $F(0)=1$. The map $F$ is a solution of the following equation:

$$
\left\{\begin{array}{l}
F(z)=(1 / \lambda) F^{p}(\lambda z) \\
F(0)=1 \\
F(z)=H\left(z^{2}\right), \text { with } H(z) \text { univalent near } 0
\end{array}\right.
$$


where $-1<\lambda<1$ is the renormalization scaling ratio and $p$ is the period of renormalization.

As a consequence of 1.1 we obtain

$$
F^{p^{m}}(z)=\lambda^{m} F\left(z / \lambda^{m}\right)
$$

whenever both sides of the equation are defined. Recall that $F$ has a maximal analytic extension $\widehat{F}: \widehat{W} \rightarrow \mathbb{C}$ to an open simply connected domain $\widehat{W} \supset \mathbb{R}$ such that $\widehat{W}$ is dense in $\mathbb{C}$ (see [20, Theorem 7.11]). Moreover, $\widehat{W} \supset \mathbb{R}$ and all critical values of $\widehat{F}$ are real. There exists a domain $W$ containing zero such that $\left.\widehat{F}\right|_{W}$ is a quadratic-like map with range equal to a slit plane $\mathbb{C} \backslash((-\infty, \alpha] \cup[\beta, \infty)) \supset W$ for some $\alpha<0, \beta>0$. In particular, $(\alpha, \beta) \supset$ $W \cap \mathbb{R}$ contains the segment $[-1,1]$. From now on we denote the quadratic-like restriction $\left.\widehat{F}\right|_{W}$ by $F$. So we assume that $F$ is not defined outside $W$.

For a set $A \subset \mathbb{C}$ and $n \in \mathbb{Z}_{+}$we denote

$$
A^{(n)}=|\lambda|^{n} A \text {. }
$$

The $n$th pre-renormalization $F_{n}$ of $F$ is the restriction of $F^{p^{n}}$ onto $W^{(n)}=$ $\lambda^{n} W$ and

$$
F_{n}(z)=\lambda^{n} F\left(z / \lambda^{n}\right)
$$

Based on sets $W^{(n)}$ we define analogues of Avila-Lyubich sets $X_{n}$ and $Y_{n}$. For each $n \in \mathbb{N}$, let $\widetilde{X}_{n}$ denote the set of points $z \in W^{(1)}$ with $F^{k}(z) \in W^{(n)}$ for some $k \geq 0$. Set

$$
\tilde{\eta}_{n}=\frac{\operatorname{area}\left(\widetilde{X}_{n}\right)}{\operatorname{area}\left(W^{(1)}\right)} .
$$

Thus, $\tilde{\eta}_{n}$ is the probability that the orbit of a point randomly chosen from $W^{(1)}$ with respect to Lebesgue measure will intersect $W^{(n)}$. By construction, $\widetilde{X}_{n+1} \subset \widetilde{X}_{n}$ for any $n$. Therefore, $\tilde{\eta}_{n}$ is non-increasing in $n$. Similarly to [8] we have:

LEMMA 3. If $\tilde{\eta}_{n}$ converges to 0 exponentially fast then so does $\eta_{n}$.

Proof. Using properties of nice domains we find that there exists $n_{0}$ such that $V^{n+n_{0}} \subset W^{(n)}$ for every $n$. Then $X_{n+n_{0}} \subset \lambda^{-1} \tilde{X}_{n+n_{0}+1}$ for every $n$, which proves the lemma.

Denote by $\widetilde{Y}_{n}$ the set of points inside $W^{(n)}$ which never return to $W^{(n)}$ under iterates of $F: \widetilde{Y}_{n}=\left\{z \in W^{(n)}: F^{k}(z) \notin W^{(n)}\right.$ for all $\left.k \in \mathbb{N}\right\}$. Set

$$
\tilde{\xi}_{n}=\frac{\operatorname{area}\left(\tilde{Y}_{n}\right)}{\operatorname{area}\left(W^{(n)}\right)} .
$$

One of the main results of the present paper is the following: 
THEOREM 4. Let $F$ be a real periodic point of renormalization. There exists a constructive constant $C$ such that for every $n, m \in \mathbb{N}$ one has

$$
\tilde{\eta}_{n+m} \leq C^{2} \tilde{\eta}_{n} \tilde{\eta}_{m+1} / \tilde{\xi}_{n}
$$

The constant $C$ is defined implicitly in Corollary 16. In Subsection 4.4 we provide a method for estimating $C$. Using Theorems 2 and 4 and Lemma 3 we obtain:

Corollary 5. If for some $n \in \mathbb{N}$ one has $C^{2} \tilde{\eta}_{n} / \tilde{\xi}_{n}<1$ then $J_{F}$ has Hausdorff dimension less than 2.

In Section 4 we present numerical results for several periodic points of renormalization with periods $3,4,5$ and 6 suggesting that the corresponding Julia sets have Hausdorff dimension less than 2.

In the proof of Theorem 4 we closely follow the approach of 8 . However, we have to make several modifications compared to the case $p=2$ studied in [8]. For instance, to prove Theorem 4 for any period $p$ we cannot use the details of the combinatorial structure of the map $F$ as in 8 , Proposition 3.1, Lemma 3.2, Proposition 3.2, and Lemma 3.3], since this structure depends significantly on the combinatorics of the critical orbit and varies substantially for different periodic points $F$ of renormalization. Instead, we give new proofs of analogous statements (e.g. Proposition 15, a generalization of [8. Proposition 4.1]) using some general properties of real periodic points of renormalization (e.g. Lemmas 10 and 11 and Proposition 12 .

2. Structure of real periodic points of renormalization. Recall that $W$ is symmetric with respect to the axes such that $F=\left.\widehat{F}\right|_{W}$ is quadratic-like with image equal to a slit plane. Let $x_{0}>0, y_{0}=i t_{0}$ with $t_{0}>0$ be such that $W^{(1)} \cap \mathbb{R}=\left[-x_{0}, x_{0}\right], W^{(1)} \cap i \mathbb{R}=\left[-y_{0}, y_{0}\right]$. Let $\mathbb{H}_{+}=\{z: \operatorname{Im} z>0\}$ be the upper half-plane, and $\mathbb{H}_{-}=\{z: \operatorname{Im} z<0\}$ the lower half-plane.

The following statement for any period $p$ can be proven in the same way as in the period-doubling case (see [12] or [3]):

THEOREM 6. All critical points of $\widehat{F}$ are simple. The critical values of $\widehat{F}$ are contained in the real axis. Moreover, for any $z \in \widehat{W}$ such that $\widehat{F}(z) \notin \mathbb{R}$, there exists a bounded open set $U(z) \ni z$ such that $\widehat{F}$ is one-to-one on $U(z)$ and $\widehat{F}(U(z))$ coincides with $\mathbb{H}_{+}$or $\mathbb{H}_{-}$.

Similarly to [3] and [8], we introduce a combinatorial partition of $\widehat{W}$.

DeFinition 7. Let $\mathcal{P}$ be the set of all connected components of $\widehat{F}^{-1}(\mathbb{C} \backslash \mathbb{R})$. For each $n \in \mathbb{Z}_{+}$, set

$$
\mathcal{P}^{(n)}=\left\{\lambda^{n} P: P \in \mathcal{P}\right\} .
$$


From 1.1 it follows that for any $m \in \mathbb{Z}_{+}$each $Q \in \mathcal{P}^{(m)}$ is mapped by $F^{p^{m}}$ bijectively onto $\mathbb{H}_{+}$or $\mathbb{H}_{-}$.

Definition 8. For $k \in \mathbb{Z}_{+}$the connected components of $\widehat{F}^{-k}(\mathbb{C} \backslash \mathbb{R})$ will be called tiles.

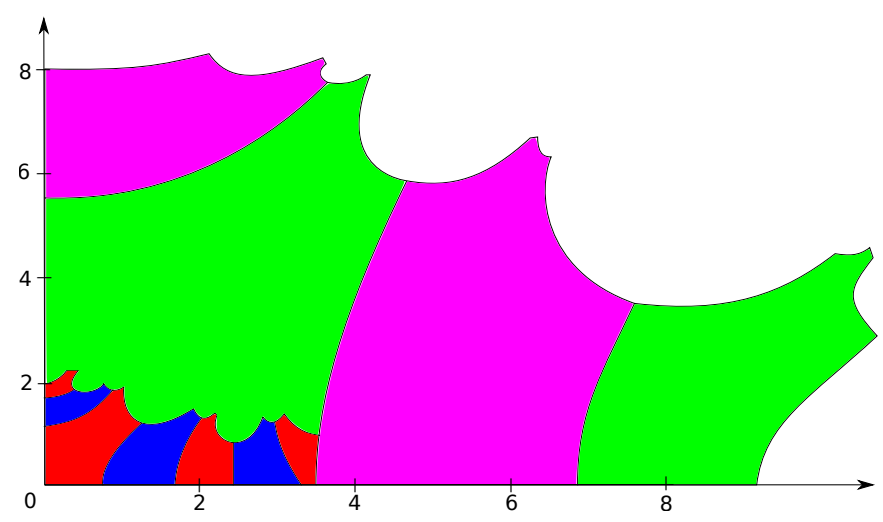

Fig. 1. Some tiles for the real period 3 fixed point of renormalization $(k=1$ and $k=2)$

Notice that any element of $\mathcal{P}^{(n)}$ is a tile for any $n \in \mathbb{Z}_{+}=\{m \in \mathbb{Z}$ : $m \geq 0\}$, as are the half-planes $\mathbb{H}_{+}$and $\mathbb{H}_{-}$. For any tile $P$, there exists $k \in \mathbb{Z}_{+}$such that the map $\widehat{F}^{k}$ sends $P$ bijectively onto $\mathbb{H}_{+}$or $\mathbb{H}_{-}$. From Theorem 6 we derive the following:

Lemma 9 (Nesting Property). For any pair of tiles $P$ and $Q$ one has $P \cap Q=\emptyset, P \subset Q$, or $Q \subset P$.

Further, for a quadrant $K \in\{$ I, II, III, IV $\}$ denote by $P_{K}$ the unique connected component of $\widehat{F}^{-1}(\mathbb{C} \backslash \mathbb{R})$ inside the quadrant $K$ such that $0 \in$ $\partial P_{K}$. Notice that the sets $P_{K}$ are tiles belonging to $\mathcal{P}=\mathcal{P}^{(0)}$. The set $W$ is equal to the interior of $\overline{P_{\mathrm{I}} \cup P_{\mathrm{II}} \cup P_{\mathrm{III}} \cup P_{\mathrm{IV}}}$. Let $J_{F}$ be the Julia set of $F$. Then $J_{F}^{(n)}=\lambda^{n} J_{F}$ is the Julia set of the pre-renormalization $F_{n}$ for every $n$.

Recall that $x_{0}>0, y_{0}=i t_{0}$ with $t_{0}>0$ are such that $W^{(1)} \cap \mathbb{R}=$ $\left[-x_{0}, x_{0}\right], W^{(1)} \cap i \mathbb{R}=\left[-y_{0}, y_{0}\right]$ and for a set $A$ we denote $A^{(n)}=\lambda^{n} A$, $n \in \mathbb{Z}_{+}$.

Lemma 10. One has $x_{0}<1$ (and so $x_{0} \in J_{F}$ ) and $\lambda y_{0} \in J_{F}$.

Proof. Assume that $x_{0} \geq 1$. Then $1=F(0) \in\left[0, x_{0}\right]$. Using the Nesting Property one can show that $F\left(P_{\mathrm{I}}^{(1)}\right) \subset P_{K}$ for $K=\mathrm{I}$ or $K=\mathrm{IV}$. Since $F^{\prime}(0)=0, F^{\prime \prime}(0)<0$ and $F$ is one-to-one on $\left[0, x_{0}\right]$, the map $F$ sends $\left[0, x_{0}\right]$ onto a segment $[a, 1]$ with $1>a \geq 0$. This implies that $F$ has an attracting fixed point on $[a, 1]$, which contradicts the renormalizability of $F$. Thus, $x_{0}<1$. 


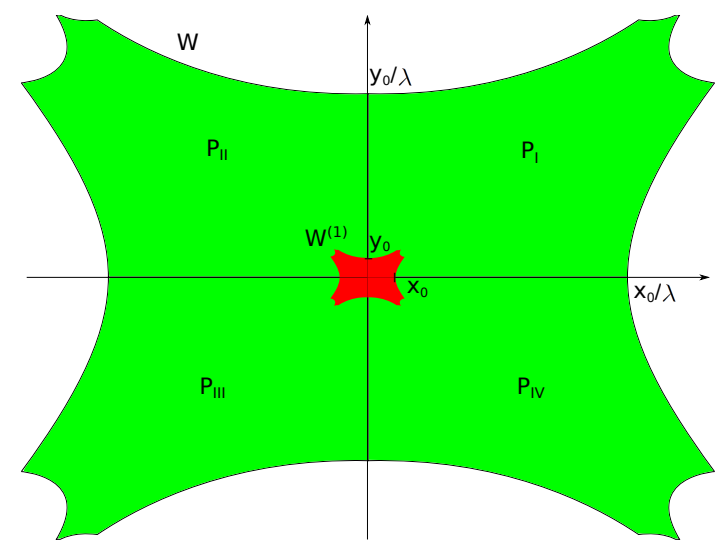

Fig. 2. Central tiles and the points $x_{0}, y_{0}$ for the real period 3 fixed point of renormalization

Further, one has $F^{p^{2}-p}\left(P_{\mathrm{I}}^{(2)}\right)=\lambda F^{p-1}\left(P_{\mathrm{I}}^{(1)}\right)$. The tile $F^{p-1}\left(P_{\mathrm{I}}^{(1)}\right)$ belongs to $\mathcal{P}$ (i.e. is mapped by $F$ one-to-one on $\mathbb{H}_{+}$or $\mathbb{H}_{-}$) and intersects $J_{F}$, therefore is equal to $P_{K}$ for some $K$. Thus, $F^{p^{2}-p}\left(P_{\mathrm{I}}^{(2)}\right)= \pm P_{K}^{(1)}$. In particular, $\left[0,|\lambda| y_{0}\right] \subset \partial P_{\mathrm{I}}^{(2)}$ is mapped by $F^{p^{2}-p}$ into $\left[-x_{0}, x_{0}\right] \subset J_{F}$. This implies that $|\lambda| y_{0} \in J_{F}$, and so $\lambda y_{0} \in J_{F}$.

Let us prove an auxiliary statement. Denote by $J_{F i}^{(n)}=F^{i}\left(J_{F}^{(n)}\right), 0 \leq$ $i<p^{n}$, the small Julia sets of the pre-renormalization $F_{n}, n \in \mathbb{Z}_{+}$.

Lemma 11. Let $P$ be a tile such that $\bar{P} \cap \mathbb{R} \neq \emptyset$ and $\bar{P} \cap J_{F, i}^{(n)} \neq \emptyset$ for some $i, n$. Then

$$
\bar{P} \cap \mathbb{R} \cap J_{F, i}^{(n)} \neq \emptyset \text {. }
$$

Proof. Assume that there exists a tile $P$ such that

$$
\bar{P} \cap \mathbb{R} \neq \emptyset, \quad \bar{P} \cap J_{F, i}^{(n)} \neq \emptyset, \quad \bar{P} \cap \mathbb{R} \cap J_{F, i}^{(n)}=\emptyset .
$$

Since $J_{F, i}^{(n)} \cap \mathbb{R} \neq \emptyset$ it is not possible that $J_{F, i}^{(n)} \subset P$. It follows that $J_{F, i}^{(n)} \cap \partial P$ $\neq \emptyset$. Therefore, $F^{k}\left(\bar{P} \cap J_{F, i}^{(n)}\right) \cap \mathbb{R} \neq \emptyset$ for some $k \in \mathbb{N}$. Fix the smallest such $k$.

Let $Q=F^{k-1}(P)$ and $\bar{Q} \cap \mathbb{R}=[a, b]$. Taking into account that

$$
\bar{Q} \cap F^{k-1}\left(J_{F, i}^{(n)}\right) \cap \mathbb{R}=\emptyset, \quad F(\bar{Q}) \cap F^{k}\left(J_{F, i}^{(n)}\right) \cap \mathbb{R} \neq \emptyset
$$

we find that $F(\partial Q \backslash \mathbb{R}) \cap \mathbb{R} \neq \emptyset$. This implies that either $a$ or $b$ is a critical point and so is zero (the unique critical point of $F$ ). Moreover, we obtain $F^{k-1}\left(\bar{P} \cap J_{F, i}^{(n)}\right) \cap i \mathbb{R} \neq \emptyset$. The latter set is a subset of $J_{F, l}^{(n)}$ where $l=i+k-1$ $\bmod p^{n}$. Since it intersects the imaginary line we have $l=0$. We conclude that

$$
0 \in F^{k-1}\left(\bar{P} \cap J_{F, i}^{(n)}\right) \cap \mathbb{R} .
$$

This contradicts the choice of $k$, finishing the proof. 
Using the Nesting Property and properties of renormalization of unimodal maps one can show:

Proposition 12.

(1) If $\left|F^{k}(0)\right| \leq|\lambda|^{n}$ for some $k, n$ then $k$ is divisible by $p^{n}$.

(2) For all $n \in \mathbb{N}$ and $1 \leq k<p^{n-1}$ one has $F^{k}\left(W^{(n)}\right) \cap J_{F}^{(n-1)}=\emptyset$.

Proof. (1) Let $k, n \in \mathbb{N}$ and $l$ be the remainder of $k$ modulo $p^{n}$. We have $F^{p^{n}}(0)=\lambda^{n} \in J_{F}^{(n)}$ and $F^{k}(0) \in J_{F, l}^{(n)}$. If $l \neq 0$ then $J_{F}^{(n)}$ may intersect $J_{F, l}^{(n)}$ only in one point, which is a periodic point of $F$. This implies that $\left|F^{k}(0)\right|>|\lambda|^{n}$.

(2) Assume that $F^{k}\left(W^{(n)}\right) \cap J_{F}^{(n-1)} \neq \emptyset$ for some $1 \leq k<p^{n-1}$. Since $F$ is real and even we have $F^{k}\left(P_{\mathrm{I}}^{(n)}\right) \cap J_{F}^{(n-1)} \neq \emptyset$. By the Nesting Property, $F^{k}\left(P_{\mathrm{I}}^{(n)}\right) \subset W^{(n-1)}$. In particular, $F^{k}(0) \in W^{(n-1)}$, and so $\left|F^{k}(0)\right| \leq\left|\lambda^{n-2}\right| x_{0}<|\lambda|^{n-2}$. By (1), $k$ is divisible by $p^{n-2}$. Taking into account (1.1), without loss of generality we may assume that $n=2$.

Further, if $p=2$ then $k=1$. We have $F\left(P_{\mathrm{I}}^{(2)}\right) \cap W^{(1)}=\emptyset$ by [9, Proposition 19]. It follows that $F\left(P_{\mathrm{I}}^{(2)}\right) \cap J_{F}^{(1)}=\emptyset$ and we obtain a contradiction.

Assume that $p>2$. Consider the small Julia sets $J_{F, i}^{(1)}=F^{i}\left(J_{F}^{(1)}\right), 0 \leq$ $i<p$. We have $F^{k}\left(P_{\mathrm{I}}^{(2)}\right) \cap J_{F}^{(1)} \neq \emptyset$. Therefore, $F^{p}\left(P_{\mathrm{I}}^{(2)}\right) \cap J_{F, p-k}^{(1)} \neq \emptyset$. By Lemma 11 .

$$
F^{p}\left({\overline{P_{\mathrm{I}}}}^{(2)}\right) \cap J_{F, p-k}^{(1)} \cap \mathbb{R} \neq \emptyset .
$$

On the other hand, $F^{p}\left(P_{\mathrm{I}}^{(2)}\right)=\lambda F\left(P_{\mathrm{I}}^{(1)}\right) \subset \lambda W=W^{(1)}$. Since $F(0)=1 \in$ $J_{F}$ it follows that $\lambda \in F^{p}\left({\overline{P_{\mathrm{I}}}}^{(2)}\right) \cap J_{F}^{(1)} \cap \mathbb{R}$.

Assume for simplicity that $F^{p}\left(P_{\mathrm{I}}^{(2)}\right)$ lies in $\{z \in \mathbb{C}: \operatorname{Re} z>0\}$. The opposite case $F^{p}\left(P_{\mathrm{I}}^{(2)}\right) \subset\{z \in \mathbb{C}: \operatorname{Re} z<0\}$ can be treated similarly. Let $\gamma$ be the minimal point of $F^{p}\left({\overline{P_{\mathrm{I}}}}^{(2)}\right) \cap J_{F, p-k}^{(1)} \cap \mathbb{R}$. Since $J_{F, p-k}^{(1)}$ and $J_{F}^{(1)}$ may intersect at most in one point we have $\gamma>|\lambda|>|\lambda| x_{0}$. It follows that $\gamma \notin{\overline{P_{\mathrm{I}}}}^{(2)}$. Since $F^{p}(z)=\lambda F(z / \lambda)$ is one-to-one on ${\overline{P_{\mathrm{I}}}}^{(1)}$ and $\gamma$ has a preimage in ${\overline{P_{\mathrm{I}}}}^{(2)}$ under $F^{p}$ we infer that $F^{p}(\gamma) \neq \gamma$.

Observe that $\gamma$ is the left end-point of the segment $I=J_{F, p-k}^{(1)} \cap \mathbb{R}$. Since $F^{p}$ preserves $J_{F, p-k}^{(1)}$ we find that $F^{p}(\gamma)$ is the right end-point of $I$. On the other hand, $F^{p}(\gamma) \in F^{2 p}\left({\overline{P_{\mathrm{I}}}}^{(2)}\right)=\lambda F^{2}\left({\overline{P_{\mathrm{I}}}}^{(1)}\right) \subset \lambda \bar{W}=\bar{W}^{(1)}$, since $p>2$. This implies that $I \subset \bar{W}^{(1)}$. However, $F^{p}$ has a critical point in the interior of $I$. We conclude that $F$ has a non-zero critical point inside $W$, which is not possible. This contradiction finishes the proof. 
3. Proof of Theorem 4. In this section we prove Theorem 4 following the approach of $[8]$.

3.1. Copies of tiles. Similarly to [8, Definition 4.1], to study the quantities $\tilde{\eta}_{n}, n \in \mathbb{N}$, we introduce copies of tiles.

Definition 13. For $k \in \mathbb{Z}_{+}$we will call a tile $Q$ a copy of the tile $P$ under $F^{k}$ if $F^{k}(Q)=P$.

For $n \in \mathbb{Z}_{+}$and a quadrant $K \in\{$ I, II, III, IV $\}$ we will call a copy $P$ of $P_{K}^{(n)}$ under $F^{k}$ primitive if $F^{j}(P) \cap W^{(n)}=\emptyset$ for all $0 \leq j<k$.

We will call a copy $P$ of $P_{K}^{(n)}$ separated if there exists $0 \leq j<k$ such that $F^{j}(P) \subset W^{(n)}$ and $F^{j}(P) \cap J_{F}^{(n-1)}=\emptyset$ for the maximal such $j$.

Let $P$ be a separated copy of $P_{K}^{(n)}$ under $F^{k}$ and $j<k$ be the maximal number such that $F^{j}(P) \subset W^{(n)}$. Then by definition for each $0 \leq i \leq j$ the set $F^{i}(P)$ is again a separated copy of $P_{K}^{(n)}$.

LEMma 14. For any separated copy $T$ of $P_{K}^{(n)}, K \in\{\mathrm{I}, \mathrm{II}, \mathrm{III}, \mathrm{IV}\}, n \in$ $\mathbb{Z}_{+}$, one has $T \cap J_{F}^{(n-1)}=\emptyset$.

Proof. Assume that there exists a separated copy $T$ of $P_{J}^{(n)}$ under $F^{k}$ such that $T \cap J_{F}^{(n-1)} \neq \emptyset$. Since $J_{F}^{(n-1)}$ is invariant under $F^{p^{n-1}}$ we deduce that $F^{l p^{n-1}}(T) \cap J_{F}^{(n-1)} \neq \emptyset$ for all $l$ for which it is defined. Let $j<k$ be the maximal number such that $F^{j}(T) \subset W^{(n)}$. Since $T$ is separated we have $F^{j}(T) \cap J_{F}^{(n-1)}=\emptyset$. Therefore, $j$ is not divisible by $p^{n-1}$. Set $l=\left[j / p^{n-1}\right]$ and $r=(l+1) p^{n-1}-j$. Then $0<r<p^{n-1}$ and

$$
F^{r}\left(W^{(n)}\right) \cap J_{F}^{(n-1)} \supset F^{r}\left(F^{j}(T)\right) \cap J_{F}^{(n-1)}=F^{(l+1) p^{n-1}}(T) \cap J_{F}^{(n-1)} \neq \emptyset .
$$

This contradicts the conclusion of Proposition 12 , and finishes the proof.

3.2. Koebe space. In this subsection we will show that iterates of $F$ corresponding to primitive and separated copies have bounded distortion.

Set $c_{\mathrm{cl}}=\min \left\{\left|F^{l}(0)\right|: 1 \leq l<p\right\} /|\lambda|$. Observe that $c_{\mathrm{cl}} \leq|F(0)| /|\lambda|=$ $1 /|\lambda|$. By Proposition $12, c_{\mathrm{cl}}>1$. The following is a generalization of [8, Proposition 4.1].

Proposition 15. Let $T$ be a primitive or separated copy of $P_{K}^{(n)}$ under $F^{k}, k \in \mathbb{Z}_{+}$. Then the inverse branch $\phi: P_{K}^{(n)} \rightarrow T$ of $F^{k}$ analytically continues to a univalent map on $\lambda^{n} \mathbb{C}_{\text {cut }}$, where

$$
\mathbb{C}_{\text {cut }}=\mathbb{C} \backslash\left(\left(-\infty, c_{\mathrm{cl}}\right] \cup\left[c_{\mathrm{cl}}, \infty\right)\right) .
$$

Proof. Assume that the statement is false. Let $T$ be primitive or separated copy of $P_{K}^{(n)}$ such that the inverse branch $F^{-k}: P_{K}^{(n)} \rightarrow T$ does not have a univalent continuation to $\lambda^{n} \mathbb{C}_{\text {cut }}$. Assume that $k$ is minimal possible 


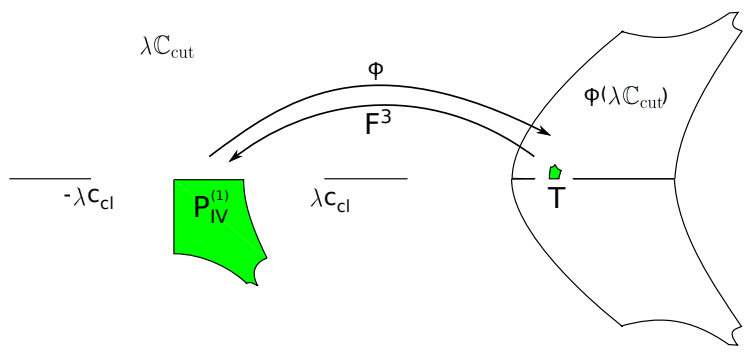

Fig. 3. An illustration to Proposition 15 Here $F$ is a period 3 real periodic point of renormalization and $T$ is a primitive copy of $P_{\mathrm{IV}}^{(1)}$ under $F^{3}$ (i.e. $p=3, n=1, k=3$, $K=\mathrm{IV})$.

with the above condition. Due to real analyticity of $F$ without loss of generality we may assume that $P_{K} \subset \mathbb{H}_{+}$, i.e. $K=\mathrm{I}$ or $K=\mathrm{II}$. Theorem 6 implies that the inverse branch $F^{-k}: P_{K}^{(n)} \rightarrow T$ admits an analytic continuation $\phi$ on $\mathbb{H}_{+}$extending to a continuous function on $\overline{\mathbb{H}_{+}}$. Moreover, the only possible reason for $\phi$ not to be extendable to an analytic function on $\lambda^{n} \mathbb{C}_{\text {cut }}$ is that $F^{k}$ has a critical point on $\phi\left(\left(-|\lambda|^{n} c_{\mathrm{cl}},|\lambda|^{n} c_{\mathrm{cl}}\right)\right)$. By minimality of $k, 0 \in \phi\left(\left(-|\lambda|^{n} c_{\mathrm{cl}},|\lambda|^{n} c_{\mathrm{cl}}\right)\right)$. Thus, we obtain $0 \in \overline{\phi\left(\mathbb{H}_{+}\right)}$and

$$
F^{k}(0) \in\left(-|\lambda|^{n} c_{\mathrm{cl}},|\lambda|^{n} c_{\mathrm{cl}}\right) \subset\left(-|\lambda|^{n-1},|\lambda|^{n-1}\right) \subset W^{(n-1)} .
$$

By Proposition $12, k$ is divisible by $p^{n-1}$.

Further, assume that $k \geq p^{n}$. Taking into account that $0 \in \overline{\phi\left(\mathbb{H}_{+}\right)}$, using the Nesting Property we obtain $T \subset \phi\left(\mathbb{H}_{+}\right) \subset W^{(n)}$. It follows that $T$ is not primitive, so it must be separated. By Lemma 14 we have $T \cap J_{F}^{(n-1)}=\emptyset$. On the other hand, since $k$ is divisible by $p^{n-1}, F^{k}$ preserves $J_{F}^{(n-1)}$. Moreover, on $J_{F}^{(n-1)}$ the map $F^{k}$ coincides with $F_{n-1}^{l}$, where $l=k / p^{n-1}$. Taking into account that $F^{k}$ is one-to-one on $\phi\left(\mathbb{H}_{+}\right)$we obtain

$$
F^{k}\left(\phi\left(\mathbb{H}_{+}\right) \cap J_{F}^{(n-1)}\right)=\mathbb{H}_{+} \cap J_{F}^{(n-1)} \quad \text { and } \quad F^{k}(T) \cap J_{F}^{(n-1)}=\emptyset .
$$

This contradicts the definition of $T$. It follows that $k<p^{n}$. Using 1.2 we obtain

$$
\left|F^{k}(0)\right|=\left|\lambda^{n-1} F^{l}(0)\right| \geq|\lambda|^{n} c_{\mathrm{cl}} .
$$

This contradicts 3.2 and finishes the proof.

Notice that [8, Proposition 4.1] concerns the situation of Proposition 15 in the case $p=2$, i.e. when $F$ is the fixed point of period-doubling renormalization. The statement obtained in 8 is slightly stronger than the result of Proposition 15 for $p=2$. Namely, for $p=2$ the set $\mathbb{C}_{\text {cut }}$ from (3.1) can be replaced by a larger set $\mathbb{C} \backslash\left((-\infty,-1 /|\lambda|) \cup\left(F(\lambda) / \lambda^{2}, \infty\right)\right)$. However, the proof of Proposition 15 is substantially simpler. The statement 
of Proposition 15 can be strengthened for $p>2$ as well, but at the cost of increased technicality. For our purposes the present statement is sufficient.

Combined with the Koebe Distortion Theorem (see e.g. [10]), Proposition 15 implies the following:

COROLlary 16. There exists a constant $C>0$ such that the following is true. Let $T$ be a primitive or separated copy of $P_{K}^{(n)}$ under $F^{k}$ for some $k \geq 0$ and $K \in\{\mathrm{I}, \mathrm{II}, \mathrm{III}, \mathrm{IV}\}$. Let $\phi: P_{K}^{(n)} \rightarrow T$ be the corresponding branch of $F^{-k}$. Then for any $x, y \in P_{J}^{(n)}$ one has

$$
\frac{\left|\phi^{\prime}(x)\right|}{\left|\phi^{\prime}(y)\right|} \leq C
$$

In particular, for any two measurable subsets $A, B$ of $P_{K}$ of positive Lebesgue measure one has

$$
\frac{\operatorname{area}\left(F^{-k}\left(B^{(n)}\right) \cap T\right)}{\operatorname{area}\left(F^{-k}\left(A^{(n)}\right) \cap T\right)} \leq C^{2} \frac{\operatorname{area}(B)}{\operatorname{area}(A)} .
$$

3.3. Recursive estimates. Fix $n, m \in \mathbb{N}$. Recall that $\widetilde{X}_{n}$ denotes the set of points $z \in W^{(1)}$ with forward orbit intersecting $W^{(n)}$ :

$$
\widetilde{X}_{n}=\left\{z \in W^{(1)}: F^{k}(z) \in W^{(n)} \text { for some } k \in \mathbb{Z}_{+}\right\} .
$$

And $\widetilde{Y}_{n}$ is the set of points inside $W^{(n)}$ which never return to $W^{(n)}$ under iterates of $F$ :

$$
\widetilde{Y}_{n}=\left\{z \in W^{(n)}: F^{k}(z) \notin W^{(n)} \text { for all } k \in \mathbb{N}\right\} .
$$

Also, we will be interested in the set of points in $W^{(n)}$ whose forward orbits under iterates of $F_{n-1}$ intersect $W^{(n+m)}$. Taking into account that $F_{n-1}(z)=$ $\lambda^{n-1} F\left(z / \lambda^{n-1}\right)$ we deduce that this set coincides with $\lambda^{n-1} \widetilde{X}_{m+1}$ :

$$
\left\{z \in W^{(n)}: F_{n-1}^{k}(z) \in W^{(n+m)} \text { for some } k \in \mathbb{Z}_{+}\right\}=\lambda^{n-1} \widetilde{X}_{m+1} .
$$

To study the sets $\widetilde{X}_{n}$ and $\widetilde{Y}_{n}$ we introduce some additional notations. For a copy $Q$ of $P_{K}^{(n)}, K \in\{\mathrm{I}, \mathrm{II}, \mathrm{II}, \mathrm{IV}\}$, under $F^{k}$ introduce the sets:

$$
Y_{Q}=Q \cap F^{-k}\left(\widetilde{Y}_{n}\right), \quad X_{Q}=Q \cap F^{-k}\left(\lambda^{n-1} \widetilde{X}_{m+1}\right) .
$$

Denote by $\mathcal{S P}=\mathcal{S P}(n)$ the set of all primitive or separated copies of sets $P_{K}^{(n)}, K \in\{$ I, II, III, IV $\}$. Let $\mathcal{S P} \mathcal{P}_{a}$ be the set of all copies $Q \in \mathcal{S P}$ such that $F^{j}(Q) \not \subset \lambda^{n-1} \widetilde{X}_{m+1}$ for all $0 \leq j<k$, where $k$ is such that $F^{k}(Q)=P_{K}^{(n)}$.

LEMma 17. The sets from the collection $\left\{Y_{Q}\right\}_{Q \in \mathcal{S} \mathcal{P}_{a}} \cup\left\{X_{T}\right\}_{T \in \mathcal{S} \mathcal{P}_{a}}$ are pairwise disjoint.

Proof. Fix a copy $Q$ of $P_{L}^{(n)}$ under $F^{j}$ and a copy $T$ of $P_{K}^{(n)}$ under $F^{k}$ such that $Q, T \in \mathcal{S P}_{a}$. Assume first that $Q \neq T$ and $Y_{Q} \cap Y_{T} \neq \emptyset$. Without loss of 
generality, let $k \geq j$. We have $F^{j}\left(Y_{Q}\right) \subset \widetilde{Y}_{n}$. Therefore, $F^{k-j}\left(\widetilde{Y}_{n}\right) \supset F^{k}\left(Y_{Q}\right)$ has a non-empty intersection with $F^{k}\left(Y_{T}\right) \subset W^{(n)}$. This contradicts the definition of $\tilde{Y}_{n}$, and so $Y_{Q}$ cannot intersect $Y_{T}$.

Assume now that $Y_{Q} \cap X_{T} \neq \emptyset$. If $j<k$ then $F^{k}\left(Y_{Q}\right) \subset F^{k-j}\left(\widetilde{Y}_{n}\right)$ cannot intersect $F^{k}\left(X_{T}\right) \subset \lambda^{n-1} \widetilde{X}_{m+1} \subset W^{(n)}$ by definition of $\widetilde{Y}_{n}$. Thus, $j \geq k$. Then $F^{k}(Q)$ has a non-empty intersection with $F^{k}(T) \subset \lambda^{n-1} \widetilde{X}_{m+1}$. This contradicts the definition of $\mathcal{S P}_{a}$. Thus, $Y_{Q}$ cannot intersect $X_{T}$.

Finally, assume that $X_{Q} \cap X_{T} \neq \emptyset$. It is not hard to see that $j \neq k$. Without loss of generality, let $j<k$. Then $F^{j}\left(X_{Q}\right) \subset \lambda^{n-1} \widetilde{X}_{m+1}$ has a non-trivial intersection with $F^{j}\left(X_{T}\right)$. It follows that

$$
F^{j}(T) \cap \lambda^{n-1} \widetilde{X}_{m+1} \neq \emptyset .
$$

Let $z$ be a point in the last intersection. Then $F_{n-1}^{s}(z) \in P_{M}^{(n+m)}$ for some $s \in \mathbb{Z}_{+}$and for some quadrant $M$. Let $R$ be the copy of $P_{M}^{(n+m)}$ under $F^{s p^{n-1}}$ containing $z$. Since $R$ and $F^{j}(T)$ are tiles, by the Nesting Property, one of them contains the other.

CASE 1: $F^{j}(T) \subset R$. Since $F_{n-1}^{s}(z)$ is well defined, we have $F^{r p^{n-1}}(z) \subset$ $W^{(n-1)}$ for $0 \leq r \leq s$. The Nesting Property implies that $F^{r p^{n-1}}(R) \subset$ $W^{(n-1)}$ for $0 \leq r \leq s$. It follows that $R$ is a connected component of $F_{n-1}^{-s}\left(P_{M}^{(n+m))}\right)$. In particular, $R \subset \lambda^{n-1} \widetilde{X}_{m+1}$. This contradicts the fact that $T \in \mathcal{S P}_{a}$.

CASE 2: $R \subset F^{j}(T)$. Since $J_{F}^{(n-1)}$ is completely invariant under $F_{n-1}$ and $J_{F}^{(n-1)} \cap P_{M}^{(n+m))} \neq \emptyset$ we have $R \cap J_{F}^{(n-1)} \neq \emptyset$. Using Lemma 14 , we find that $T$ is not a separated copy. However, $T$ is not a primitive copy either, since $F^{j}(T) \subset W^{(n)}$. This contradicts $T \in \mathcal{S P}_{a}$ and finishes the proof.

LEMMA 18. The set

$$
\widetilde{X}_{n+m} \backslash \bigcup_{Q \in \mathcal{S} \mathcal{P}_{a}} X_{Q}
$$

consists of a countable collection of analytic curves.

Proof. Let $z \in \widetilde{X}_{n+m}$. Observe that $W^{(n+m)} \subset \lambda^{n-1} \widetilde{X}_{m+1}$. Therefore, there exists $k \in \mathbb{Z}_{+}$such $F^{k}(z) \in \lambda^{n-1} \widetilde{X}_{m+1}$. Let $k$ be the minimal such number. Assume that $F^{k}(z) \notin \mathbb{R} \cup i \mathbb{R}$. Let $K$ be the quadrant containing $F^{k}(z)$ and let $Q$ be the copy of $P_{K}^{(n)}$ under $F^{k}$ containing $z$. Let us show that $Q \in \mathcal{S P}_{a}$.

Assume that $Q$ is not primitive. Then there exists $0 \leq j<k$ such that $F^{j}(Q) \subset W^{(n)}$. Let $j$ be the maximal such number. Let $F^{j}(Q) \cap J_{F}^{(n-1)} \neq \emptyset$. Taking into account that $F^{k}(Q) \cap J_{F}^{(n-1)} \neq \emptyset$ and any two distinct small Julia sets $J_{F, i}^{(n-1)}, 0 \leq i<p^{n-1}$, may intersect only in a real point, we infer 
that $k-j$ is divisible by $p^{n-1}$. Let $l=(k-j) / p^{n-1}$. Since $\left.F^{k-j}\right|_{J_{F}^{n-1}}=$ $\left.F_{n-1}^{l}\right|_{J_{F}^{n-1}}$, using the Nesting Property we can conclude that $F_{n-1}^{l}$ is well defined on $F^{j}(Q)$, and in particular at $F^{j}(z)$. We find that $F^{j}(z) \in \lambda^{n-1} \widetilde{X}_{m+1}$, which contradicts $k>j \geq 0$. Thus, $F^{j}(Q) \cap J_{F}^{(n-1)}=\emptyset$ and $Q$ is separated.

We have shown that $Q \in \mathcal{S P}$. By minimality of $k$, we have $F^{j}(z) \notin$ $\lambda^{n-1} \widetilde{X}_{m+1}$ for all $0 \leq j<k$. It follows that $Q \in \mathcal{S P}_{a}$, which finishes the proof.

Recall that $\tilde{\eta}_{n}=\operatorname{area}\left(\widetilde{X}_{n}\right) / \operatorname{area}\left(W^{(1)}\right), \tilde{\xi}_{n}=\operatorname{area}\left(\widetilde{Y}_{n}\right) / \operatorname{area}\left(W^{(n)}\right)$.

Proof of Theorem 4. Let $C$ be the constant from Corollary 16. Let $n, m \in \mathbb{N}$. We need to show that

$$
\tilde{\eta}_{n+m} \leq C^{2} \tilde{\eta}_{n} \tilde{\eta}_{m+1} / \tilde{\xi}_{n} .
$$

By Lemma 18, modulo a countable set of analytic curves, the set $\widetilde{X}_{n+m}$ is the union of all sets of the form $X_{Q}, Q \in \mathcal{S} \mathcal{P}_{a}$. Using Corollary 16 and the fact that the sets $\widetilde{X}_{k}, \widetilde{Y}_{k}, k \in \mathbb{N}$, are symmetric with respect to the coordinate axes, we obtain

$$
\frac{\operatorname{area}\left(X_{Q}\right)}{\operatorname{area}\left(Y_{Q}\right)} \leq C^{2} \frac{\operatorname{area}\left(\lambda^{n-1} \widetilde{X}_{m+1}\right)}{\operatorname{area}\left(\widetilde{Y}_{n}\right)}=C^{2} \frac{\tilde{\eta}_{m+1}}{\tilde{\xi}_{n}} .
$$

Using Lemma 17 we arrive at

$$
\operatorname{area}\left(\widetilde{X}_{n+m}\right)=\sum_{Q \in \mathcal{S P} a} \operatorname{area}\left(X_{Q}\right) \leq C^{2} \frac{\tilde{\eta}_{m+1}}{\tilde{\xi}_{n}} \sum_{Q \in \mathcal{S P} \mathcal{P}_{a}} \operatorname{area}\left(Y_{Q}\right) .
$$

Since the union of the sets $X_{Q}$ and $Y_{Q}$ over $Q \in \mathcal{S P} \mathcal{P}_{a}$ is a subset of $\widetilde{X}_{n}$, we obtain

$$
\operatorname{area}\left(\widetilde{X}_{n+m}\right) \leq C^{2} \frac{\tilde{\eta}_{m+1}}{\tilde{\xi}_{n}} \operatorname{area}\left(\widetilde{X}_{n}\right) .
$$

This finishes the proof.

4. Numerical results and algorithms. In this section we present numerical results for several real periodic points of Feigenbaum renormalization of periods $3,4,5$ and 6 . We compute bounds on $\tilde{\eta}_{n}, \tilde{\xi}_{n}$ for $n=2$ or $n=3$ and on the constant $C$ from Corollary 16 . Also, we sketch some algorithms we have used to compute the above quantities.

Notice that a real periodic point $F$ of renormalization of period $p$ (in the sense of (1.1) ) is uniquely determined by a permutation $s$ of the set $\{0,1, \ldots, p-1\}$ decoding how the first $p$ elements $0, F(0), \ldots, F^{p-1}(0)$ of the critical orbit are permuted by ordering them increasingly, i.e. $F^{j}(0)<F^{k}(0)$ for $0 \leq j, k \leq p-1$ if and only if $s(j)<s(k)$. Since the kneading sequence of $F$ can be reconstructed from $s$, for any permutation $s$ of $\{0,1, \ldots, p-1\}$ 
there exists at most one real periodic point $F_{s}$ of Feigenbaum renormalization corresponding to $s$. We encode a permutation $s$ by the sequence $\left[s^{-1}(0), s^{-1}(1), \ldots, s^{-1}(p-1)\right]$ in which each number $i$ is on position $s(i)$. We obtain the following empirical results:

- For $F_{[1,0,2]}: \tilde{\eta}_{3}<0.0105, \tilde{\xi}_{3}>0.69, C^{2}<52$.

- For $F_{[1,0,3,2]}: \tilde{\eta}_{3}<0.01, \tilde{\xi}_{3}>0.3, C^{2}<26$.

- For $F_{[1,0,4,3,2]}: \tilde{\eta}_{2}<0.014, \tilde{\xi}_{2}>0.42, C^{2}<10.23$.

- For $F_{[2,0,4,3,1]}: \tilde{\eta}_{2}<0.014, \tilde{\xi}_{2}>0.43, C^{2}<22.2$.

- For $F_{[1,0,5,4,3,2]}: \tilde{\eta}_{2}<0.08, \tilde{\xi}_{2}>0.6, C^{2}<3.1$.

In each case we find (modulo numerical errors and errors coming from using an approximation of $F$ ) that $C^{2} \tilde{\eta}_{n} / \tilde{\xi}_{n}<1$. By Corollary 5 , this suggests that the corresponding Julia sets have Hausdorff dimension less than 2. Below we briefly describe some algorithms we have used in the computations. Notice that approximations of the sets $P_{\mathrm{I}}, \widetilde{X}_{n}$, and $\widetilde{Y}_{n}$, as well as an approximation of the constant $C$, can be obtained similarly to the case of the fixed point of period-doubling renormalization [8].

\subsection{Computing real periodic points of Feigenbaum renormal-} ization. Notice that there exist effective algorithms for computing certain fixed points of renormalization (see e.g. [5], [15], and [16]). Here we have used a more straightforward algorithm, which is less technical, easier to implement in a programming language, and easier to adjust to any real periodic point of renormalization.

Given a period $p$ we start by computing all real superattracting parameters $c$ of period $p$, i.e. $c \in \mathbb{R}$ such that $P_{c}^{p}(0)=0$ and $P_{c}^{j}(0) \neq 0$ for all $1 \leq j \leq p-1$, where $P_{c}(z)=z^{2}+c$. For each such parameter $c$ there is a bifurcation cascade starting at the hyperbolic component of the Mandelbrot set containing $c$. The limit of this cascade is a Feigenbaum parameter (denoted by $l(c)$ ) with a stationary combinatorics. An approximation of this parameter can be found as follows.

Given a unimodal map $f(z)$ with critical point at 0 , the kneading sequence is the sequence $K(f)=\left\{k_{1}, k_{2}, \ldots\right\} \subset\{-1,0,1\}^{\mathbb{N}}$, where $k_{i}=-1$ if $f^{i}(0)<0, k_{i}=1$ if $f^{i}(0)>0$ and $k_{i}=0$ if $f^{i}(0)=0$. Let $s$ be the permutation of $\{0, \ldots, p-1\}$ induced by $P_{c}$ as described above. Taking into account that $P_{l(c)}^{p}$ is conjugate to $P_{l(c)}$ on a domain containing the postcritical set we can compute the kneading sequence $K\left(P_{l(c)}\right)=\left[i_{1}, i_{2}, \ldots\right]$ of $P_{l(c)}$. Further, we start computing an approximation of $l(c)$ with a segment $I_{0}=[-2,-1]$ containing $l(c)$. At step $n$, given a segment $I_{n}$ containing $l(c)$ we subdivide it into two subsegments by the midpoint $m_{n}$ of $I_{n}$. Comparing the kneading sequences $K\left(P_{m_{n}}\right)$ and $K\left(P_{l(c)}\right)$ we find to which subsegment $l(c)$ belongs 
and denote this subsegment by $I_{n+1}$. Since the length of $I_{n}$ is $2^{-n}$ this allows us to obtain an arbitrarily good approximation of $l(c)$.

Recall that the iterates of the renormalization $\mathcal{R}^{k}\left(P_{l(c)}\right)$ converge to a periodic point $F$ of renormalization. Given a sufficiently good approximation $\tilde{l}$ of $l(c)$ we approximate $F$ as $\mathcal{R}^{k}\left(P_{\tilde{l}}\right)$ with appropriately chosen $k$. In more detail, we introduce the norms on the space of convergent power series at zero:

$$
\left\|\sum_{k=0}^{\infty} a_{k} z^{k}\right\|_{r}=\sum_{k=0}^{\infty}\left|a_{k}\right| r^{k}, \quad r>0 .
$$

We choose $r$ smaller than the radius of convergence of $F$. Then we find the smallest $k$ such that

$$
\left\|\mathcal{R}^{k+1}\left(P_{\tilde{l}}\right)-\mathcal{R}^{k}\left(P_{\tilde{l}}\right)\right\|_{r}>\left\|\mathcal{R}^{k}\left(P_{\tilde{l}}\right)-\mathcal{R}^{k-1}\left(P_{\tilde{l}}\right)\right\|_{r}
$$

and set $\mathcal{R}^{k}\left(P_{\tilde{l}}\right)$ to be the approximation $\widetilde{F}$ of $F$.

To improve the approximation we consider the family

$$
\widetilde{F}_{\tau}(z)=\widetilde{F}(z)+\left(\tau-\frac{1}{2} \widetilde{F}^{\prime \prime}(0)\right) z^{2}
$$

of maps having the same Taylor coefficients at zero as $\widetilde{F}$ except the coefficient at $z^{2}$ equal to $\tau$. This family is complete. Therefore, there exists $\tau$ for which $\widetilde{F}_{\tau}$ belongs to the stable manifold of $F$ under the map $\mathcal{R}$. We find a sufficiently good approximation $\tilde{\tau}$ of $\tau$ using the same approach as for finding the approximation $\tilde{l}$ of $l(c)$. Then we compute $\mathcal{R}^{k}\left(\widetilde{F}_{\tilde{\tau}}\right)$ for appropriately chosen $k$. We repeat the above two steps until we get an approximation $\widetilde{F}$ of $F$ with sufficiently small $\|\mathcal{R} \widetilde{F}-\widetilde{F}\|_{r}$.

4.2. Estimating the central tiles. Given a real periodic point $F$ of renormalization we approximate the central tile $P_{\mathrm{I}}$ as follows. Take a sufficiently large rectangle of the form $\{z \in \mathbb{C}: 0<\operatorname{Re} z<a, 0<\operatorname{Im} z<b\}$. Take a sufficiently dense finite subset $\mathcal{C}$ of this rectangle. By the Koebe Quarter Theorem, for any point $z \in \mathcal{C}$ the disk $U_{z}$ of radius

$$
\frac{|\operatorname{Re}(F(z))|}{4\left|F^{\prime}(z)\right|}
$$

belongs to $F^{-1}(\mathbb{C} \backslash \mathbb{R})$. Set

$$
\mathcal{U}=\bigcup_{z \in \mathcal{C}} U_{z} .
$$

The connected component of $\mathcal{U}$ closest to the origin is an approximation of $P_{\mathrm{I}}$ from below.

4.3. Estimating the sets $\widetilde{X}_{n}$. Since $\widetilde{X}_{n}$ is symmetric with respect to the coordinate axes we can restrict our attention to the first quadrant. Take a sufficiently dense finite subset $\mathcal{C}$ in $P_{\mathrm{I}}^{(1)}$. Fix a sufficiently large $N \in \mathbb{N}$. Given a point $z \in \mathcal{C}$ approximate the iterates $z_{j}=F^{j}(z)$ and the derivatives 
$D F^{j}(z), 0 \leq j \leq N$. If $z_{j} \notin W^{(n)}$ for $0 \leq j \leq N$, set $U_{z}=\emptyset$. Assuming $z_{j} \in W^{(n)}$ for some $0 \leq j \leq N$ let $j$ be minimal possible with this property, and let $R$ be the radius of the largest disk centered at $z_{j}$ which is contained in $W^{(n)}$. Then, by the Koebe Quarter Theorem, the disk $U_{z}$ of radius $\frac{R}{4\left|D F^{j}(z)\right|}$ centered at $z$ belongs to $\widetilde{X}_{n}$. We approximate $\widetilde{X}_{n} \cap P_{\mathrm{I}}^{(1)}$ by $\bigcup_{z \in \mathcal{C}} U_{z}$.

An approximation of $\widetilde{Y}_{n}$ can be obtained in a similar way.

4.4. Estimating the distortion constant $C$. To estimate $C$ from Corollary 16 following [8] consider a univalent function $\phi: \mathbb{C}_{\text {cut }} \rightarrow \mathbb{C}$. Let $w \in \mathbb{C}_{\text {cut }}$. There is a unique conformal isomorphism $H_{w}: \mathbb{C}_{\text {cut }} \rightarrow \mathbb{D}$ such that $H_{w}(w)=0$ and $H_{w}^{\prime}(w)>0$. The function $\varphi=\phi \circ H_{w}^{-1}$ is univalent on the unit disk $\mathbb{D}$. Using the Koebe Distortion Theorem we obtain, for every $z \in \mathbb{C}_{\text {cut }}$,

$$
\frac{\left|\phi^{\prime}(z)\right|}{\left|\phi^{\prime}(w)\right|} \leq C(z, w):=\frac{1+\left|H_{w}(z)\right|}{\left(1-\left|H_{w}(z)\right|\right)^{3}} \frac{\left|H_{w}^{\prime}(z)\right|}{\left|H_{w}^{\prime}(w)\right|} .
$$

We estimate $C:=\sup \left\{C(z, w): z, w \in P_{\mathrm{I}}\right\}$ by taking the maximum of $C(z, w)$ over a finite grid of points sufficiently dense in $P_{\mathrm{I}}$. Notice that these estimates can be made rigorous (see [8] for details).

Acknowledgements. The author is grateful to Feliks Przytycki for valuable comments.

This work was partially supported by the National Science Centre, Poland, grant 2016/23/P/ST1/04088 under POLONEZ programme which has received funding from the EU Horizon 2020 research and innovation programme under the MSCA grant agreement No. 665778.

\section{References}

[1] A. Avila and M. Lyubich, Hausdorff dimension and conformal measures of Feigenbaum Julia sets, J. Amer. Math. Soc. 21 (2008), 305-383.

[2] A. Avila and M. Lyubich, Lebesgue measure of Feigenbaum Julia sets, arXiv:1504.02986 (2015).

[3] X. Buff, Geometry of the Feigenbaum map, Conform. Geom. Dynam. 3 (1999), 79-101.

[4] X. Buff and A. Cheritat, Quadratic Julia sets with positive area, Ann. of Math. 172 (2012), 637-746.

[5] A. Burbanks, A. Osbaldestin, and J. Thurlby, Rigorous computer-assisted bounds on the period doubling renormalisation fixed point and eigenfunctions in maps with critical point of degree 4, arXiv:2006.13127 (2020).

[6] P. Coullet et C. Tresser, Itération d'endomorphismes et groupe de renormalisation, J. Phys. Colloque 39 (1978), no. C5, 25-28.

[7] A. Douady and J. Hubbard, On the dynamics of polynomial-like maps, Ann. Sci. École Norm. Sup. 18 (1985), 287-344.

[8] A. Dudko and S. Sutherland, On the Lebesgue measure of the Feigenbaum Julia set, Invent. Math. 221 (2020), 167-202. 
[9] A. Dudko and M. Yampolsky, Poly-time computability of the Feigenbaum Julia set, Ergodic Theory Dynam. Systems 36 (2016), 2441-2462.

[10] P. L. Duren, Univalent Functions, Springer, 1983.

[11] H. Epstein, Fixed points of composition operators II, Nonlinearity 2 (1989), 305-310.

[12] H. Epstein, Fixed points of the period-doubling operator, lecture notes, Lausanne, 1992.

[13] M. Feigenbaum, Quantitative universality for a class of class of non-linear transformations, J. Statist. Phys. 19 (1978), 25-52.

[14] M. Feigenbaum, The universal metric properties of non-linear transformations, J. Statist. Phys. 21 (1979), 669-706.

[15] P. Hertling and C. Spandl, Computing a solution of Feigenbaum's functional equation in polynomial time, Logical Methods Computer Sci. 10 (2014), no. 4, 1-9.

[16] O. Lanford III, A computer-assisted proof of the Feigenbaum conjectures, Bull. Amer. Math. Soc. (N.S.) 6 (1982), 427-434.

[17] M. Lyubich, Dynamics of quadratic polynomials, I-II, Acta Math. 178 (1997), 185297.

[18] M. Lyubich, Feigenbaum-Coullet-Tresser universality and Milnor's hairiness conjecture, Ann. of Math. 149 (1999), 319-420.

[19] W. de Melo and S. van Strien, One-Dimensional Dynamics, Springer, 1993.

[20] C. McMullen, Renormalization and 3-manifolds which Fiber over the Circle, Ann. of Math. Stud. 142, Princeton Univ. Press, 1996.

[21] D. Sullivan, Bounds, quadratic differentials, and renormalization conjectures, in: Mathematics into Twenty-First Century, Amer. Math. Soc., 1992, 417-466.

Artem Dudko

Institute of Mathematics

Polish Academy of Sciences

Śniadeckich 8

00-656 Warszawa, Poland

ORCID: 0000-0003-2706-3215

E-mail: adudko@impan.pl 